International Mathematical Forum, 1, 2006, no. 30, 1473 - 1482

\title{
On numerical solutions of a superlinear elliptic problem
}

\author{
G. A. Afrouzi \\ Department of Mathematics, Faculty of Basic Sciences \\ Mazandaran University, Babolsar, Iran \\ e-mail: afrouzi@umz.ac.ir \\ S. Khademloo and A. Yazdani \\ Department of Mathematics, Faculty of Basic Sciences \\ Mazandaran University, Babolsar, Iran
}

\begin{abstract}
In this work we consider numerical positive solutions of the equation $-\Delta u=\lambda f(u)$ with Dirichlet boundary condition in a bounded domain $\Omega$, where $\lambda>0$ and $f(u)$ is a superlinear function of $u$. We study the behavior of the branches of numerical positive solutions for varying $\lambda$.
\end{abstract}

Mathematics Subject Classification: 35J60, 35B30, 65N99

Keywords: Elliptic boundary value problems, multiple solutions, finite difference method, interpolation formula.

\section{Introduction}

We are interested in the positive solutions of the problem

$$
\begin{cases}-\Delta u(x)=\lambda f(x, u(x)) & x \in \Omega \\ u(x)=0 & x \in \partial \Omega\end{cases}
$$

where $\Omega$ is a bounded domain in $\mathbf{R}^{N}(N \geq 3)$ with boundary $\partial \Omega$, and $f(u)=a u-b u^{2}+c u^{3}(a, b, c>0)$.

The problems involving Laplace equation arise quite frequently in the biological, social and physical sciences. For example solutions of $-\Delta u=\lambda f(u)$ correspond to steady states for time motion, with $f$ corresponding to extermal driving forces. The Laplace equation also plays an important role in field theories in which a field (e.g. electric, magnetic gravitation forces, or fluid velocity 
field) is given as the gradient of a potential function $u$ ([8]).

On the other hand the Dirichlet boundary value condition has important physical significance. In electrostatics, for example, this condition specified the value of the potential function $u$ on $\partial \Omega$ which induces the electric field $\vec{E}=-\nabla u$ in $\Omega$. If we can show the equation $-\Delta u=\lambda f(u)$ with Dirichlet condition is well-posed, this means that the electric field is completely determined by the charge distribution inside $\Omega$ together with the value of the potential function $u$ on $\partial \Omega$.

In this paper we study numerical solutions of the equation (1) that arises in wide fields of physics, and so it has been studied by several authors. Among others it describes problems of thermal self-ignition [3], diffusion phenomena induced by nonlinear sources [5] or a ball of isothermal gas in gravitational equilibrium as proposed by Lord Kelvin [1]. We also refer to [4,9] where different models and further references may be found. In this paper we concentrate on the numerical positive solutions of temperature distribution in an object heated by the application of a uniform electric current suggested in [6]. In fact we show that the first eigenvalue of the problem

$$
\begin{cases}-\Delta u(x)=\lambda a u(x) & x \in \Omega \\ u(x)=0 & x \in \partial \Omega .\end{cases}
$$

is a bifurcation point of the branch of numerical solutions and so there is another branch of solutions that for any positive $\lambda$ admits a numerical solution. The outline of the paper is following.

In the next section we present a useful numerical method and introduce the framework of the procedure to find numerical solutions. Section 3 contains some numerical results of the problem (1) for varying $\lambda$ and $a, b, c$.

\section{Finite difference method}

Numerical techniques based on finite difference schemes can lead us to obtain approximate solutions for any PFEs $([2,7,10])$. In particular for an Elliptic partial differntial equation of second order such as

$$
L u=\sum \sum\left(-a_{i j} \frac{\partial^{2} u}{\partial x_{i} \partial x_{j}}\right)+\sum b_{i} \frac{\partial u}{\partial x_{i}}+c u=f(\lambda, u),
$$

we can use this technique. In fact the main idea is finding a numerical solution for (1) in bounded domain $\Omega$ that in special points of $\Omega$ is adapted with exact solution, i.e., we seek a solution array $\mathbf{u}$ that in any points of a discrete grid $\Omega \subset \Omega, \mathbf{u}(x)=u(x)$ where $u$ is the exact solution of (1). It is often 
advantageous to study derivatives using the limit of difference quotients, for this reason we define "forward difference quotient" as follows:

$$
\Delta_{i}^{+} u(x)=\frac{u\left(x+h e_{i}\right)-u(x)}{h}
$$

where $h \neq 0, e_{i}$ denotes the unit vector in the $x_{i}$-direction, $h$ is small enough that $0<|h|<\operatorname{dist}(x, \partial \Omega)$.

Moreover we can define " backward difference quotient":

$$
\Delta_{i}^{-} u(x)=\frac{u(x)-u\left(x-h e_{i}\right)}{h}
$$

If we put together these quotients, we find a better approximation for $\frac{\partial u(x)}{\partial x_{i}}$ as follows:

$$
\delta_{i} u(x)=\frac{u\left(x+h e_{i}\right)-u\left(x-h e_{i}\right)}{2 h} .
$$

It is proved in [8] that if we consider $u \in \mathrm{C}^{1}(\Omega)$ then

$$
\delta_{i} u(x) \longrightarrow \frac{\partial u(x)}{\partial x_{i}} \quad \text { as } h \rightarrow 0 .
$$

We can continue this procedure to gain higher derivatives such as

$$
\delta_{i i} u(x)=\frac{u\left(x+h e_{i}\right)-2 u(x)+u\left(x-h e_{i}\right)}{h^{2}}
$$

and

$$
\begin{gathered}
\delta_{i j} u(x)= \\
\frac{u\left(x+h e_{i}+k e_{j}\right)-u\left(x+h e_{i}-k e_{j}\right)-u\left(x-h e_{i}+k e_{j}\right)+u\left(x-h e_{i}-k e_{j}\right)}{4 h k}
\end{gathered}
$$

that tends to

$$
\frac{\partial^{2} u(x)}{\partial x_{i}^{2}}, \quad \frac{\partial^{2} u(x)}{\partial x_{i} \partial x_{j}}
$$

as $h \rightarrow 0$ and $|(h, k)| \rightarrow 0$ respectively.

Now by replacing these approximations in any elliptic equation of second order we arrive at a finite system of equations that after solving we can obtain desired array $\mathbf{u}$.

The method of differences is especially suitable for the solution of boundary value problems, for instance, the problem of determining a function that satisfies the Laplace equation in the interior of a given field $\Omega$ and possesses given values at the boundary of the field; such problems arise in the exploration of stationary temperature distribution when the temperature at the boundary of the field is known, in investigating the tension in a twisted rod of prismetic section, etc. In this cases the procedure is as above.

It is remained that how we can choose the points of grid. If $\Omega$ is a regular 
domain for example a square in plane, i.e., $\Omega=[a, b] \times[c, d]$. We can suppose the solution domain of the problem is covered by a mesh of grid-lines

$$
\begin{array}{ll}
x_{i}=a+i h & i=0,1,2, \cdots, n_{1}, \\
y_{j}=c+j k & j=0,1,2, \cdots, n_{2}
\end{array}
$$

parallel to the axes and

$$
x_{0}=a, \quad x_{n_{1}}=b, \quad y_{0}=c, \quad y_{n_{2}}=d .
$$

Approximations $u_{i j}$ to $u\left(x_{i}, y_{j}\right)$ are calculated at the point of intersection of these lines, namely, $\left(x_{i}, y_{j}\right)$ which is referred to as the $(i, j)$ grid-point. The constant spatial and temporal grid-spacing are

$$
h=\frac{(b-a)}{n_{1}}, \quad k=\frac{(d-c)}{n_{2}}
$$

respectively.

But a large number of physical problems have irregular boundary. For investigation a point like $P$ near bound of domain that does not have distance equal to $h$ with boundary, can be used some techniques. A precise technique, is using of interpolation. In this section we explain it briefly.

For simplicity we limit us in two dimension space. Suppose the points of grid $\Omega$ that has distances equal $h$ and $k$ with $P$ in directions $x$ and $y$ be $P_{1}$ and $P_{2}$ and the nearest point to $P$ on the boundary in direction $x$ and $y$ be $Q_{1}$ and $Q_{2}$ respectively. Because of Dirichlet boundary condition we know $u\left(Q_{1}\right)=u\left(Q_{2}\right)=0$.

Let $\operatorname{dist}\left(P, Q_{1}\right)=d_{1}$ and $\operatorname{dist}\left(P, Q_{2}\right)=d_{2}$ where $0 \leq d_{1}, d_{2} \leq 1$. We want to find $\frac{\partial u}{\partial x}(P)$ and $\frac{\partial u}{\partial y}(P)$ and $\frac{\partial^{2} u}{\partial x^{2}}(P)$ and $\frac{\partial^{2} u}{\partial y^{2}}(P)$ simultaneously.

By using Taylor expansion around $P$ we get $u(x, y)=$

$u(P)+x\left(\frac{\partial u}{\partial y}\right)(P)+y\left(\frac{\partial u}{\partial y}\right)(P)+\frac{x^{2}}{2}\left(\frac{\partial^{2} u}{\partial x^{2}}\right)(P)+x y\left(\frac{\partial^{2} u}{\partial x \partial y}\right)(P)+\frac{y^{2}}{2}\left(\frac{\partial^{2} u}{\partial y^{2}}\right)(P)+\cdots$.

Without loss of generality we can suppose $P$ is $(0,0)$ and so we can write points $P_{1}, P_{2}, Q_{1}$ and $Q_{2}$ such as:

$$
(-h, 0), \quad(0,-k), \quad\left(d_{1} h, 0\right), \quad\left(0, d_{2} k\right)
$$

respectively.

After substitution last formulas in Taylor expansion and omission $o\left(h^{3}\right)$ and $o\left(k^{3}\right)$, we arrive at: 


$$
\begin{cases}d_{2} k u_{y}(P)+\frac{1}{2}\left(d_{2} k\right)^{2} u_{y y}(P) & =u\left(Q_{2}\right)-u(P) \\ d_{1} h u_{x}(P)+\frac{1}{2}\left(d_{1} h\right)^{2} u_{x x}(P) & =u\left(Q_{1}\right)-u(P) \\ -k u_{y}(P)+\frac{1}{2} k^{2} u_{y y}(P) & =u\left(P_{2}\right)-u(P) \\ -h u_{x}(P)+\frac{1}{2} h^{2} u_{x x}(P) & =u\left(P_{1}\right)-u(P)\end{cases}
$$

that gives

$$
\begin{aligned}
& \frac{\partial u}{\partial x}(P)=h^{-1}\left[\frac{u\left(Q_{1}\right)}{d_{1}\left(1+d_{1}\right)}-\frac{d_{1} u\left(P_{1}\right)}{\left(1+d_{1}\right)}-\frac{\left(1-d_{1}\right) u(P)}{d_{1}}\right]+o\left(h^{2}\right) \\
& \frac{\partial^{2} u}{\partial x^{2}}(P)=2 h^{-2}\left[\frac{u\left(Q_{1}\right)}{d_{1}\left(1+d_{1}\right)}-\frac{u\left(P_{1}\right)}{\left(1+d_{1}\right)}-\frac{u(P)}{d_{1}}\right]+o(h) \\
& \frac{\partial u}{\partial y}(P)=k^{-1}\left[\frac{u\left(Q_{2}\right)}{d_{2}\left(1+d_{2}\right)}-\frac{d_{2} u\left(P_{2}\right)}{\left(1+d_{2}\right)}-\frac{\left(1-d_{2}\right) u(P)}{d_{2}}\right]+o\left(k^{2}\right) \\
& \frac{\partial^{2} u}{\partial y^{2}}(P)=2 k^{-2}\left[\frac{u\left(Q_{2}\right)}{d_{1}\left(1+d_{1}\right)}-\frac{u\left(P_{2}\right)}{\left(1+d_{2}\right)}-\frac{u(P)}{d_{2}}\right]+o(k) .
\end{aligned}
$$

If the points $P_{1}$ and $P_{2}$ lie irregularly, we can use this procedure for them again. To find the value of $u$ in the point $P$ we apply linear interpolation formula in direction $x$ as follows

$$
u(P)=\left(\frac{d_{1}}{1+d_{1}}\right) u\left(P_{1}\right)+\left(\frac{1}{1+d_{1}}\right) u\left(Q_{1}\right)
$$

or in direction $y$ as follows

$$
u(P)=\left(\frac{d_{2}}{1+d_{2}}\right) u\left(P_{2}\right)+\left(\frac{1}{1+d_{2}}\right) u\left(Q_{2}\right) .
$$

This technique is one of the useful methods that developed from the work of Gerschgarin, G and Z. Angew.

\section{$3 \quad$ Numerical Results}

In this section we consider problem (1) and use all of discussions in previous section to find numerical solutions.

At first we note that to solve problem (1) we consider $N \geq 3$. Let $N=3$ and $\Omega=[0,1] \times[0,1] \times[0,1]$ and the grid $\Omega \subset \Omega$ be a division of $\Omega$ and 
$h=\frac{1}{4}\left(n_{1}=n_{2}=n_{3}=4\right)$.

We solve numerically the problem

$$
\begin{cases}-\left(u_{x x}+u_{y y}+u_{z z}\right)=\lambda\left[u(x, y, z)-3 u(x, y, z)^{2}+u(x, y, z)^{3}\right] & (x, y, z) \in \Omega \\ u(x, y, z)=0 & (x, y, z) \in \partial \Omega\end{cases}
$$

, i.e., $a=c=1$ and $b=3$. Dirichlet boundary condition lead us to have

$$
u_{0, j, k}=u_{i, 0, k}=u_{i, j, 0}=0 \quad \forall 1 \leq i, j, k \leq 3
$$

where $u_{i, j, k}=u\left(x_{i}, y_{j}, z_{k}\right)$.

By using the approximation of $u_{x x}$ and $u_{y y}$ and $u_{z z}$ we have a system of equations of this type

$$
\begin{gathered}
-\frac{u_{i+1, j, k}+u_{i-1, j, k}+u_{i, j+1, k}+u_{i, j-1, k}+u_{i, j, k+1}+u_{i, j, k-1}-6 u_{i, j, k}}{h^{2}}= \\
\lambda\left(u_{i, j, k}-3 u_{i, j, k}^{2}+u_{i, j, k}^{3}\right) .
\end{gathered}
$$

Some of the equations of this system mentioned follows:

$$
\begin{array}{ll}
16\left(u_{211}+u_{121} u_{112}-6 u_{111}\right)+\lambda\left(u_{111}-3 u_{111}^{2}+u_{111}^{3}\right)=0 & \text { for } i=j=k=1 \\
16\left(u_{212}+u_{122}+u_{113}+u_{121}-6 u_{112}\right)+\lambda\left(u_{112}-3 u_{112}^{2}+u_{112}^{3}\right)=0 & \text { for } i=j=1, k=2
\end{array}
$$

After solving this system we can obtain $\mathbf{u}$ in grid $\Omega$ that guide us to understand the behavior of solution branches. We express just some of values of $u_{i j k} \mathrm{~s}$ in the following tables. It is easy to see that $\lambda^{+}$(the first eigenvalue of the problem (2)) in this case is 1409.3 with decimal accuracy.

Note that the first table is for the branch of solution bifurcates from $\lambda^{+}$and the second is for another branch of solutions. We mention them by $\mathbf{u}$ and $\mathbf{w}$. 


\begin{tabular}{c||ccc}
$\lambda$ & \multicolumn{3}{c}{$\mathbf{u}$} \\
\hline \multirow{2}{*}{1} & $u_{111}=2.26 \times 10^{-13}$ & $u_{121}=4.06 \times 10^{-13}$ & $u_{131}=2.27 \times 10^{-13}$ \\
& $u_{211}=3.19 \times 10^{-13}$ & $u_{221}=5.39 \times 10^{-13}$ & $u_{231}=3.21 \times 10^{-13}$ \\
& $u_{311}=1.87 \times 10^{-13}$ & $u_{321}=3.06 \times 10^{-13}$ & $u_{331}=1.90 \times 10^{-13}$ \\
\hline \multirow{2}{*}{25} & $u_{111}=2.10 \times 10^{-12}$ & $u_{121}=3.22 \times 10^{-12}$ & $u_{131}=2.11 \times 10^{-12}$ \\
& $u_{211}=2.75 \times 10^{-12}$ & $u_{221}=4.04 \times 10^{-12}$ & $u_{231}=2.77 \times 10^{-12}$ \\
& $u_{311}=1.84 \times 10^{-12}$ & $u_{321}=2.67 \times 10^{-12}$ & $u_{331}=1.87 \times 10^{-12}$ \\
\hline \multirow{3}{*}{26.6} & $u_{111}=3.41 \times 10^{-8}$ & $u_{121}=5.16 \times 10^{-8}$ & $u_{131}=3.42 \times 10^{-8}$ \\
& $u_{211}=4.46 \times 10^{-8}$ & $u_{221}=6.46 \times 10^{-8}$ & $u_{231}=4.50 \times 10^{-8}$ \\
& $u_{311}=3.03 \times 10^{-8}$ & $u_{321}=4.35 \times 10^{-8}$ & $u_{331}=3.07 \times 10^{-8}$ \\
\hline \multirow{3}{*}{26.7} & $u_{111}=0.00045$ & $u_{121}=0.00068$ & $u_{131}=0.00045$ \\
& $u_{211}=0.00059$ & $u_{221}=0.00085$ & $u_{231}=0.00059$ \\
& $u_{311}=0.00040$ & $u_{321}=0.00057$ & $u_{331}=0.00040$ \\
\hline \multirow{3}{*}{50} & $u_{111}=0.1123$ & $u_{121}=0.1482$ & $u_{131}=0.1124$ \\
& $u_{211}=0.1402$ & $u_{221}=0.1790$ & $u_{231}=0.1405$ \\
& $u_{311}=0.1086$ & $u_{321}=0.1394$ & $u_{331}=0.1091$ \\
\hline \multirow{2}{*}{100} & $u_{111}=0.2159$ & $u_{121}=0.2540$ & $u_{131}=0.2159$ \\
& $u_{211}=0.2493$ & $u_{221}=0.2881$ & $u_{231}=0.2494$ \\
& $u_{311}=0.2145$ & $u_{321}=0.2491$ & $u_{331}=0.2146$ \\
\hline \multirow{3}{*}{1000} & $u_{111}=0.3610$ & $u_{121}=0.3678$ & $u_{131}=0.3610$ \\
& $u_{211}=0.3677$ & $u_{221}=0.3744$ & $u_{231}=0.3677$ \\
& $u_{311}=0.3610$ & $u_{321}=0.3677$ & $u_{331}=0.3610$ \\
\hline \multirow{2}{*}{1000} & $u_{111}=0.3798$ & $u_{121}=0.3805$ & $u_{131}=0.3798$ \\
& $u_{211}=0.3805$ & $u_{221}=0.3812$ & $u_{231}=0.3805$ \\
& $u_{311}=0.3798$ & $u_{321}=0.3805$ & $u_{331}=0.3798$ \\
& & &
\end{tabular}

\begin{tabular}{c||ccc}
$\lambda$ & \multicolumn{3}{|c}{$\mathbf{w}$} \\
\hline \multirow{2}{*}{$10^{-3}$} & $w_{111}=289.29$ & $w_{121}=268.896$ & $w_{131}=289.29$ \\
& $w_{211}=267.244$ & $w_{221}=244.326$ & $w_{231}=267.244$ \\
& $w_{311}=289.458$ & $w_{321}=267.229$ & $w_{331}=289.461$ \\
\hline \multirow{3}{*}{1} & $w_{111}=46.9683$ & $w_{121}=46.8729$ & $w_{131}=46.9683$ \\
& $w_{211}=46.8728$ & $w_{221}=46.7773$ & $w_{231}=46.8728$ \\
& $w_{311}=46.9683$ & $w_{321}=46.8728$ & $w_{331}=46.9683$ \\
\hline \multirow{2}{*}{10} & $w_{111}=23.9161$ & $w_{121}=23.8967$ & $w_{131}=23.9161$ \\
& $w_{211}=23.8967$ & $w_{221}=23.8774$ & $w_{231}=23.8967$ \\
& $w_{311}=23.9161$ & $w_{321}=23.8967$ & $w_{331}=23.9161$ \\
\hline \multirow{2}{*}{100} & $w_{111}=2.81949$ & $w_{121}=2.7553$ & $w_{131}=2.81949$ \\
& $w_{211}=2.75322$ & $w_{221}=2.68209$ & $w_{231}=2.75322$ \\
& $w_{311}=2.81958$ & $w_{321}=2.75322$ & $w_{331}=2.81958$ \\
\hline \multirow{2}{*}{10000} & $w_{111}=2.62018$ & $w_{121}=2.61946$ & $w_{131}=2.62018$ \\
& $w_{211}=2.61946$ & $w_{221}=2.61875$ & $w_{231}=2.61946$ \\
& $w_{311}=2.62018$ & $w_{321}=2.61946$ & $w_{331}=2.62018$
\end{tabular}


So according to the above tables we guess that $\lambda^{+}$is around 26.7 that before it we don't have have any nonzero minimal solutions.

Now we compare the numerical results for constants $a=c=1$ and $b=3$ with $a=2, b=5$ and $c=4$ that is mentioned in the following tables that show $\lambda^{+}$ in this case is around 13.4.

\begin{tabular}{c||ccc}
$\lambda$ & \multicolumn{3}{c}{$\mathbf{u}$} \\
\hline \multirow{2}{*}{1} & $u_{111}=3.74 \times 10^{-12}$ & $u_{121}=6.66 \times 10^{-12}$ & $u_{131}=3.75 \times 10^{-12}$ \\
& $u_{211}=5.24 \times 10^{-12}$ & $u_{221}=8.82 \times 10^{-12}$ & $u_{231}=5.28 \times 10^{-12}$ \\
& $u_{311}=3.09 \times 10^{-12}$ & $u_{321}=5.04 \times 10^{-12}$ & $u_{331}=3.14 \times 10^{-12}$ \\
\hline \multirow{2}{*}{25} & $u_{111}=1.12 \times 10^{-9}$ & $u_{121}=1.81 \times 10^{-9}$ & $u_{131}=1.12 \times 10^{-9}$ \\
& $u_{211}=1.46 \times 10^{-9}$ & $u_{221}=2.26 \times 10^{-9}$ & $u_{231}=1.48 \times 10^{-9}$ \\
& $u_{311}=9.36 \times 10^{-10}$ & $u_{321}=1.40 \times 10^{-9}$ & $u_{331}=9.53 \times 10^{-10}$ \\
\hline \multirow{3}{*}{13.3} & $u_{111}=7.28 \times 10^{-7}$ & $u_{121}=1.10 \times 10^{-6}$ & $u_{131}=7.31 \times 10^{-7}$ \\
& $u_{211}=9.53 \times 10^{-7}$ & $u_{221}=1.38 \times 10^{-6}$ & $u_{231}=9.60 \times 10^{-7}$ \\
& $u_{311}=6.48 \times 10^{-7}$ & $u_{321}=9.28 \times 10^{-7}$ & $u_{331}=6.56 \times 10^{-7}$ \\
\hline \multirow{3}{*}{13.4} & $u_{111}=0.00137$ & $u_{121}=0.002069$ & $u_{131}=0.00137$ \\
& $u_{211}=0.00179$ & $u_{221}=0.0025$ & $u_{231}=0.00180$ \\
& $u_{311}=0.00122$ & $u_{321}=0.00174$ & $u_{331}=0.00123$ \\
\hline \multirow{2}{*}{50} & $u_{111}=0.353546$ & $u_{121}=0.44405$ & $u_{131}=0.353634$ \\
& $u_{211}=0.423983$ & $u_{221}=0.52511$ & $u_{231}=0.424252$ \\
& $u_{311}=0.346441$ & $u_{321}=0.422426$ & $u_{331}=0.346979$ \\
\hline \multirow{2}{*}{100} & $u_{111}=0.492698$ & $u_{121}=0.489763$ & $u_{131}=0.491913$ \\
& $u_{211}=0.483215$ & $u_{221}=0.455413$ & $u_{231}=0.480927$ \\
& $u_{311}=0.482602$ & $u_{321}=0.465202$ & $u_{331}=0.480903$ \\
\hline \multirow{3}{*}{10000} & $u_{111}=0.643676$ & $u_{121}=0.642885$ & $u_{131}=0.643676$ \\
& $u_{211}=0.642884$ & $u_{221}=0.642091$ & $u_{231}=0.642884$ \\
& $u_{311}=0.643676$ & $u_{321}=0.642884$ & $u_{331}=0.643676$ \\
& & &
\end{tabular}




\begin{tabular}{c||ccc}
$\lambda$ & \multicolumn{3}{c}{$\mathbf{w}$} \\
\hline \multirow{2}{*}{$10^{-3}$} & $w_{111}=110.311$ & $w_{121}=108.105$ & $w_{131}=110.154$ \\
& $w_{211}=113.88$ & $w_{221}=51.273$ & $w_{231}=114.002$ \\
& $w_{311}=105.25$ & $w_{321}=114.798$ & $w_{331}=107.295$ \\
\hline \multirow{2}{*}{1} & $w_{111}=46.1596$ & $w_{121}=46.1361$ & $w_{131}=46.1596$ \\
& $w_{211}=46.136$ & $w_{221}=46.1125$ & $w_{231}=46.136$ \\
& $w_{311}=46.1596$ & $w_{321}=46.136$ & $w_{331}=46.1596$ \\
\hline \multirow{3}{*}{10} & $w_{111}=23.2667$ & $w_{121}=23.262$ & $w_{131}=23.2667$ \\
& $w_{211}=23.262$ & $w_{221}=23.2573$ & $w_{231}=23.262$ \\
& $w_{311}=23.266$ & $w_{321}=23.262$ & $w_{331}=23.2667$ \\
\hline \multirow{2}{*}{100} & $w_{111}=0.9469$ & $w_{121}=0.90435$ & $w_{131}=0.9469$ \\
& $w_{211}=0.9023$ & $w_{221}=0.8549$ & $w_{231}=0.9023$ \\
& $w_{311}=0.9471$ & $w_{321}=0.9023$ & $w_{331}=0.9471$ \\
\hline \multirow{2}{*}{10000} & $w_{111}=0.81782$ & $w_{121}=0.81738$ & $w_{131}=0.81782$ \\
& $w_{211}=0.8173$ & $w_{221}=0.8169$ & $w_{231}=0.81738$ \\
& $w_{311}=0.8178$ & $w_{321}=0.8173$ & $w_{331}=0.8178$
\end{tabular}

So by using the results in these tables we can draw the bifurcation diagram of the solutions in the plane $(\lambda,\|u\|)$, where

$$
\|u\|=\|u\|_{\infty}=\sup _{(x, y, z) \in[0,1] \times[0,1] \times[0,1]} u(x, y, z)
$$

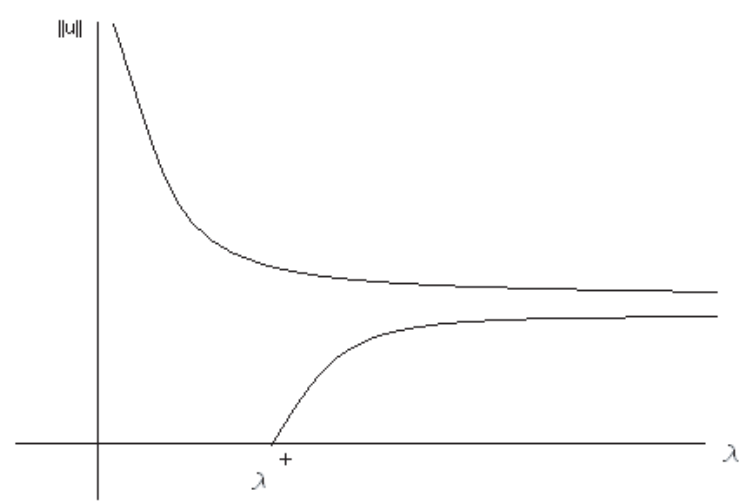

Figure 1: Bifurcation diagram 


\section{References}

[1] S. Chandrasekhar, An introduction to the study of Steller structure, Dover publications, Inc.: New York, N.Y., 1957; ii+509 pp.

[2] M. Dehghan, Numerical procedures for a boundary value problem with a non-linear boundary condition, Applied mathematics and computation 147 (2004), 291 - 306.

[3] I. M. Gelfand, Some problems in the theory of quasi-linear equations, Amer. Math. Soc. Trans., 29 (2) (1963), 295 - 381.

[4] D. D. Joseoh, T. S. Landgren, Quasilinear Dirichlet problems driven by positive sources, Arch. Rat. Mech. Anal., 49 (1973), 241 - 269.

[5] D. D. Joseoh, E .M. Sparrow, Nonlinear diffusion induced by nonlinear sources, Quart. Appl. Math., 28 (1970), 327 - 342.

[6] H. B. Keller, D. S. Cohen, Some positive problems suggested by nonlinearheat generation, J. Math. Mech., 16 (1967), 1361 - 1376.

[7] O. A. Ladyzhenskaya, The boundary value problems of mathematical physics, Springer-Verlag, New York, 1985.

[8] R. McOwen, Partial differential equations, Prentice-Hall, Inc. 1996.

[9] F. Mignot, J. P. Puel, Sur une classede problemes non lineairesavec nonlinearite positive, Croissante, Convexe. Comm. Part. Diff. Eq., 5 (8) (1980) $791-836$.

[10] I. G. Petrovsky, Lectures on partial differential equations, Dover publications, Inc. 1991.

Received: Nov. 26, 2005 